\title{
Infrared Communications for Small Spacecraft: from a Wireless Bus to Cluster Concepts
}

\author{
Suzanne C. Webb*, Wolfger Schneider, M.Ann G. Darrin, Bradley G. Boone \\ Johns Hopkins University Applied Physics Laboratory \\ Philip J. Luers ${ }^{* *}$ \\ NASA Goddard Space Flight Center
}

\begin{abstract}
Nanosatellites operating singly or in clusters are anticipated for future space science missions. To implement this new communications paradigm, we are approaching cluster communications by first developing an infrared (IR) intra-craft wireless bus capability, following initially the MIL-STD-1553B protocol. Benefits of an IR wireless bus are low mass, size, power, and cost, simplicity of implementation, ease of use, minimum EMI, and efficient and reliable data transfer. Our goals are to maximize the reliable link margin in order to afford greater flexibility in receiver placement, which will ease technology insertion. We have developed a concept demonstration using a high-speed visible-band silicon PIN photodiode and a high-efficiency visible LED operating at a data rate up to $4 \mathrm{Mb} / \mathrm{sec}$. In designing an internal IR wireless bus, we have characterized various candidate materials, emitters, and geometries, assuming a single reflection. Thus, we have measured the bidirectional reflectance distribution function (BRDF) for five different materials characteristic of typical spacecraft structures, which range from nearly Lambertian to highly specular. We have fit our data to empirical BRDF functions and modeled the detected irradiance anywhere in the plane of incidence for a divergent (LED) emitter. We have also determined the angular limits on the link geometry to remain within the required bit error rate by determining the received signal-to-noise ratio (SNR) for minimum values of irradiance received at the detector.
\end{abstract}

Keywords: infrared, communications, spacecraft, nanosats, BRDF, wireless bus, IrDA, MIL-STD-1553B

* suzanne.webb@ihuapl.edu; phone 1-443-778-6818; fax 1-443-778-6779; Johns Hopkins University Applied Physics Laboratory, Johns Hopkins Road, Laurel, MD, USA 20723-6099; **Philip.J.Luers@gsfc.nasa.gov; phone 1-301-286-5777; fax 1-301-286-5475; NASA Goddard Space Flight Center, Code 561, Greenbelt, MD, USA 2077 I

\section{INTRODUCTION}

The space community has expressed a strong interest in developing nanosatellites for use in future space science and near-earth-orbit (NEO) support functions. As the size of the satellites continues to decrease, it may be necessary to develop a new intra-satellite communications system. An infrared (IR) wireless data bus would provide efficient and reliable data transfer, while reducing the mass, size, and power requirements of a satellite. It would also have low electromagnetic interference (EMI), and minimize the need for high-cost custom electronics. An IR wireless data bus can be readily designed to transmit data at rates of at least $4 \mathrm{Mb} / \mathrm{sec}$.

Instead of working with a standard point-to-point communications system, we propose to use a diffuse reflecting surface within the satellite to broadcast the signal over the entire plane of receivers. In order to study the feasibility of implementing this communications link, it is necessary to characterize and compare various emitters, reflector materials, and geometries. Because most systems are expected to involve at least one reflection, we have measured the bidirectional reflectance distribution function (BRDF) for five candidate materials that are typically found in spacecraft. These range from nearly Lambertian to highly specular surfaces. We then fit the measured data to empirical functions describing the BRDF, and incorporate these functions into a radiometric model that calculates the magnitude and distribution of the irradiance received in the plane of incidence. By varying the different components, this model can be used to optimize an optical intrasatellite communications system.

Currently, communication buses within satellites require relatively large, heavy, custom-designed wire harnesses. The alternative of simply using fiber optic cables for a physical link is labor-intensive, because each fiber end must be 
cleaved, polished, and connectorized. These disadvantages can be eliminated by replacing the harnesses and fiber optic cables with free-space optical communication links, which would consist of compatible infrared transmitters and receivers. Working in the infrared provides a mature, commercial-off-the-shelf (COTS) technology. Wireless infrared intra-satellite communication systems offer reduced power consumption, less contribution to spacecraft mass, virtually no cabling complexity, and provide a means of efficient and reliable data transfer.

As described later in this paper, we have implemented an infrared (IR) free space communications system utilizing the MIL-STD-1553B protocol, to allow different components within the satellite to communicate with each other. In order to ensure that each transmitter can talk with each receiver, the signal is directed towards a common surface in the satellite, which then reflects the signal over the plane of the receivers. A general diagram of the layout is shown in Figure 1 . The received irradiance $\left(\mathrm{mW} / \mathrm{cm}^{2}\right)$ depends on the output power and beam profile of the emitter, the characteristics of the reflector material, the geometry of the satellite, and the location of the detector. Therefore, in order to investigate the feasibility of implementing an IR intra-satellite communications system, we have constructed a radiometric model that calculates the irradiance received by each detector, based upon the emitter and reflector characteristics, as well as the physical layout of the system. Each of these components can be varied to optimize an optical communications link.

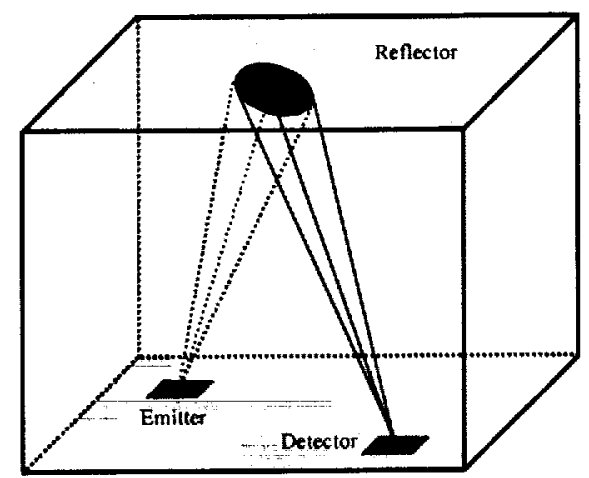

Figure 1. General layout for IR communications system.

\section{RADIOMETRIC MEASUREMENTS AND ANALYSIS}

\subsection{Bidirectional Reflectance Distribution Function}

One important factor in designing an intra-satellite optical communications link is the material used for the reflective surface. There are several candidate materials that are commonly used in satellites; however, they each have distinct reflection properties that have a significant impact upon the distribution of the reflected signal. In order to evaluate a particular material, we measure its bidirectional reflectance distribution function (BRDF) in the plane of incidence, and derive a mathematical expression that describes the scatter for all angles of incidence and reflection. These measurements are then incorporated into the model.

A scatterometer is used to determine the BRDF of a material [Ref. 1]. A diagram of the experimental setup is shown in Figure 2. The detector is mounted on one end of a motorized swing arm, and the other end of the arm is fixed at the sample. The scatterometer measures the incremental angular dependent scatter over the back hemisphere. For this set of experiments, we use an unpolarized $\mathrm{HeNe}$ laser operating at $632.8 \mathrm{~nm}$, and a large area silicon detector with a spectral bandpass filter. All measurements are made in the plane of incidence from $\theta_{\mathrm{r}}=-90^{\circ}$ to $90^{\circ}$.

Here we assume that the BRDF is the same for all $\phi_{r}$ for a particular $\theta_{r}$, which implies that the reflectance is rotationally symmetric about the specular direction. This is a good assumption for most surfaces with a roughness scale small with respect to the beam footprint. This geometry is illustrated in Figure 3, where the reflective surface lies in the xy plane, and the light is incident from the z-direction. As the sample is rotated from normal incidence to oblique angles, the illuminating spot changes from a circle to an ellipse, with its long axis in the plane of incidence. The reflectance from this elliptical spot is no longer independent of $\phi_{r}$. However, this effect is minimal for small angles of incidence $\left(\theta_{i}<45^{\circ}\right)$. 


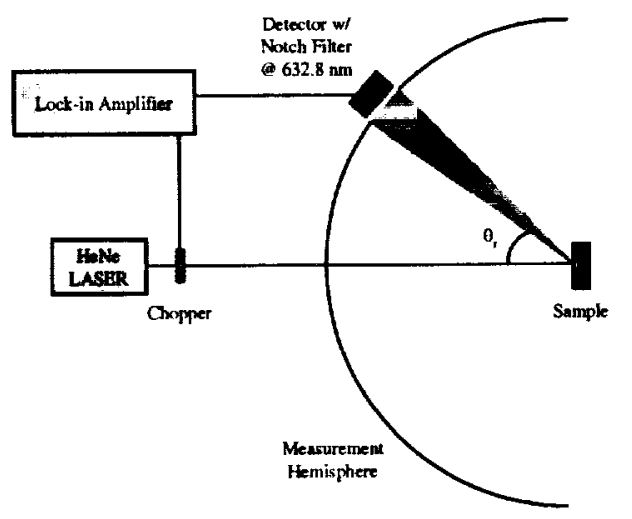

Figure 2. Experimental setup used to measure BRDF.

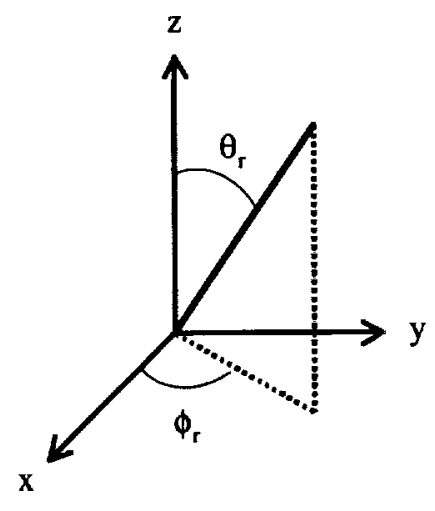

Figure 3. Geometry used for BRDF measurements.

In order to compensate for physical limitations of the experiment and derive a general expression for the BRDF as a function of the angles of incidence and reflection, the measured BRDF data are fit to an empirical formula. The measured BRDF can be expressed as the product of the total integrated reflectance, $\operatorname{TIR}\left(\theta_{i}\right)$, and a normalized phase function, $P\left(\theta_{r}, \theta_{i}\right)$ :

$$
\operatorname{BRDF}\left(\theta_{r}, \theta_{i}\right)=\operatorname{TIR}\left(\theta_{i}\right) \mathrm{P}\left(\theta_{r}, \theta_{i}\right)
$$

More specifically, the BRDF can be separated into specular and diffuse components. A specular reflection is at a specific angle from a very smooth surface $\left(\theta_{\mathrm{r}}=-\theta_{\mathrm{i}}\right)$, while a diffuse reflection is scattered at different angles from a rough surface. $A$ completely diffuse, or Lambertian, phase function is independent of the angle of incidence, and is given by:

$$
P_{\text {Lam }}\left(\theta_{\mathrm{r}}\right)=\frac{1}{\pi} \cos \theta_{\mathrm{r}}
$$

Meanwhile, the partially diffuse portion of the BRDF can be modeled with a Lorentz-type phase function as follows:

$$
P_{\text {Diff }}\left(\theta_{r}, \theta_{i}, n, \alpha\right)=\frac{n}{\pi 6.3 \alpha} \frac{\left(\frac{\alpha}{\cos \theta_{i}}\right)^{n-1}}{\left(\theta_{r}+\theta_{i}\right)^{n}+\left(\frac{\alpha}{\cos \theta_{i}}\right)^{n}} \cos \theta_{r},
$$

where $\alpha$ and $n$ are free parameters that can be varied to fit the data. Finally, because the laser source has a finite beam width and divergence, the measured specular component depends on the beam profile of the laser. After scanning across the incident beam, the distribution is fit to the following Gaussian function:

$$
P_{\text {Spec }}\left(\theta_{r}, \theta_{i}\right)=A e^{-\frac{\left(\theta_{r}+\theta_{1}\right)^{2}}{b^{2}}}
$$

A comparison of the functional forms of each BRDF phase function is shown in Figure 4.

For each sample, the BRDF is measured with several different angles of incidence. The parameters and weighting coefficients of the phase functions are then adjusted until their linear combination best approximates the measured data for each angle of incidence. For example, the measured BRDF of an aluminum sample is fit to the following empirical function:

$$
\operatorname{BRDF}_{\mathrm{Al}}\left(\theta_{\mathrm{r}}, \theta_{\mathrm{i}}\right)=0.45 \mathrm{P}_{\mathrm{Diff}}\left(\theta_{\mathrm{r}}, \theta_{\mathrm{i}}, 2,0.16\right)+0.08 \mathrm{P}_{1 \mathrm{sm}}\left(\theta_{\mathrm{r}}\right)
$$




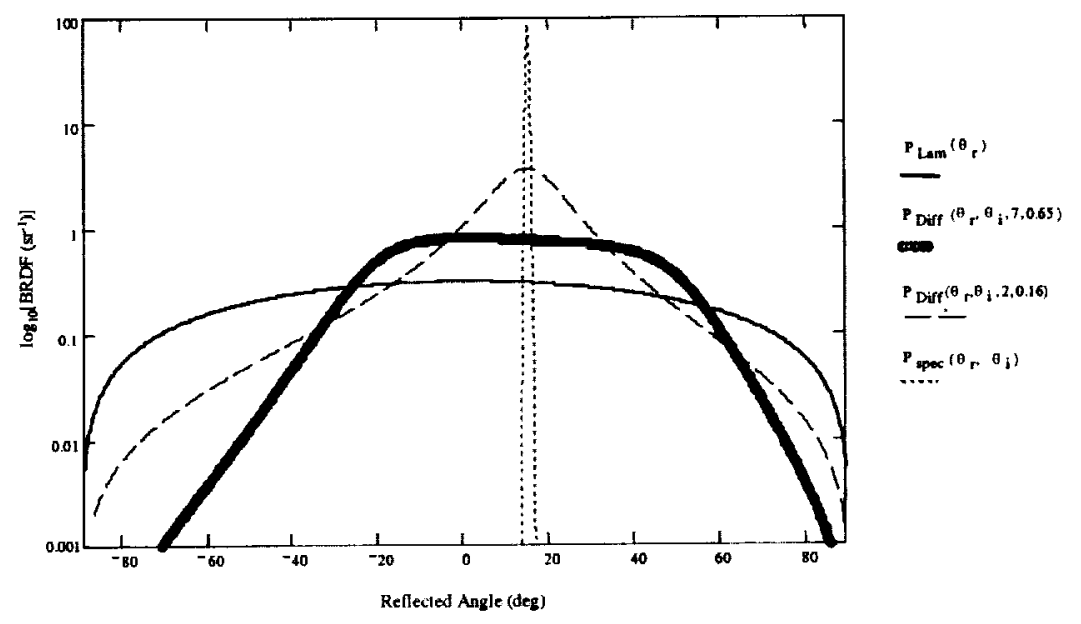

Figure 4. Functions fit to BRDF measurements $\left(\theta_{i}=-15^{\circ}\right)$.

The modeled BRDF can consist of a linear combination of any or all of the phase functions, each of which must be normalized. The total integrated reflectance (TIR) is then found by integrating the modeled BRDF over the entire hemisphere for a particular angle of incidence.

BRDF measurements and analysis have been performed on samples of five materials that are commonly found in satellites: aluminum, ceramic, delrin, kovar, and a solar cell. Figure 5 compares the BRDF for each sample, as well as the theoretical BRDF from a purely Lambertian material. In Figure 5(a-e), the BRDF is measured at an incident angle of $-15^{\circ}$, and the empirical fit is shown for each sample. This figure indicates that a solar cell has a highly specular reflectance, while the ceramic sample is nearly Lambertian. The aluminum, delrin, and kovar samples fall between these two extremes. Each plot also indicates the TIR for the sample. Figure $5 \mathrm{f}$ compares a sandblasted aluminum sample with a perfectly Lambertian surface. Because the Lambertian surface is idealized, its TIR is equal to unity.

\subsection{Model of Intra-Satellite Communications}

One spacecraft of particular interest for IR communications is a nanosatellite, which is expected to be instrumental in future space science and near-earth-orbit support functions. A conceptual diagram of a nanosatellite [Ref. 2] is shown in Figure 6. For this model, the plane of emitters and receivers will be located on the bottom interior surface, and the reflector material will be on the top interior surface. The initial guidelines set the diameter at $30 \mathrm{~cm}$ and the height at $10 \mathrm{~cm}$. Figure 7 includes the relevant parameters for the model. Here $\psi$ is the full-width beam emission angle subtended by the source, and $\theta_{\mathrm{r}}$ is the angle of reflection, as measured from the optical axis of the emitter. Each transceiver contains both an emitter and a detector, which allows a signal to be transmitted from and to any spatial location via the broadcast system. For the purposes of this model, the angle of incidence $\left(\theta_{i}\right)$ is constrained to be $0^{\circ}$ for each emitter.

In order to calculate the irradiance received at each possible detector location, we first determine the flux incident on the reflector surface. The flux from an emitter with a non-zero beam divergence can be expressed as:

$$
\Phi=2 \pi \mathrm{I}_{\mathrm{e}}\left[1-\cos \left(\frac{\psi}{2}\right)\right]
$$

where $I_{e}$ is the radiant intensity of the emitter. Note that the total flux does not depend on the angle of incidence or the distance between the planes. However, because the beam profile is Gaussian, the flux is unevenly distributed across the reflector surface. Therefore, the total flux must be multiplied by a normalized Gaussian phase function $\left(\mathrm{P}_{\text {Gauss }}\right)$ that is defined by the extent of the beam area on the reflector. The radiant intensity from the reflector is now given by:

$$
\mathrm{I}_{\text {refl }}=\Phi \cdot \mathrm{P}_{\text {Gauss }} \cdot \mathrm{BRDF}
$$




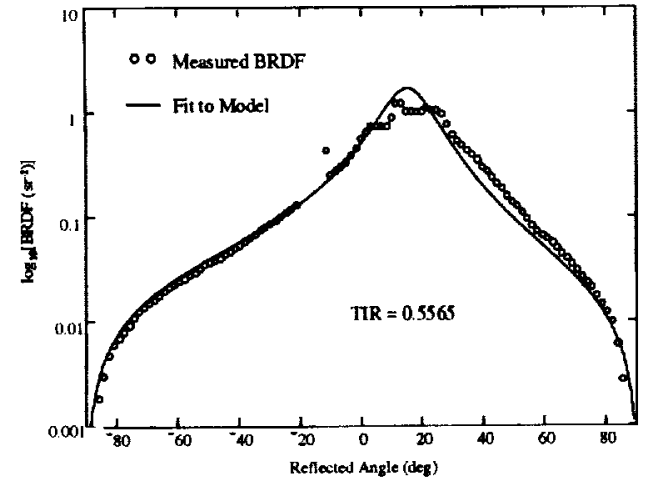

(a) Aluminum sample.

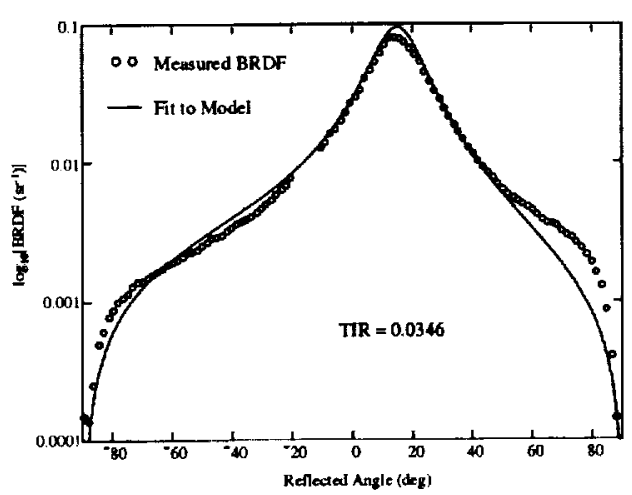

(c) Delrin sample.

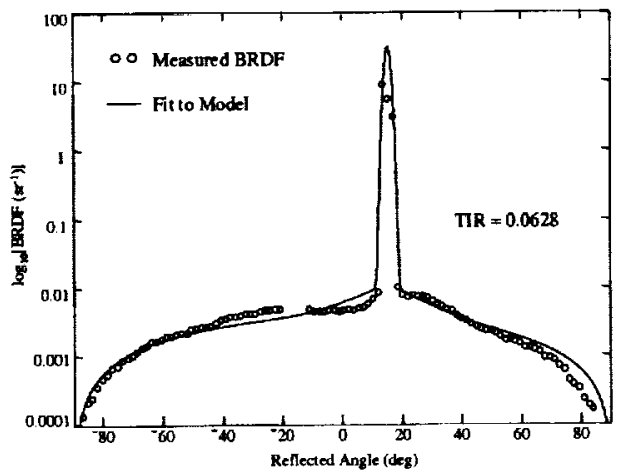

(e) Solar cell sample.

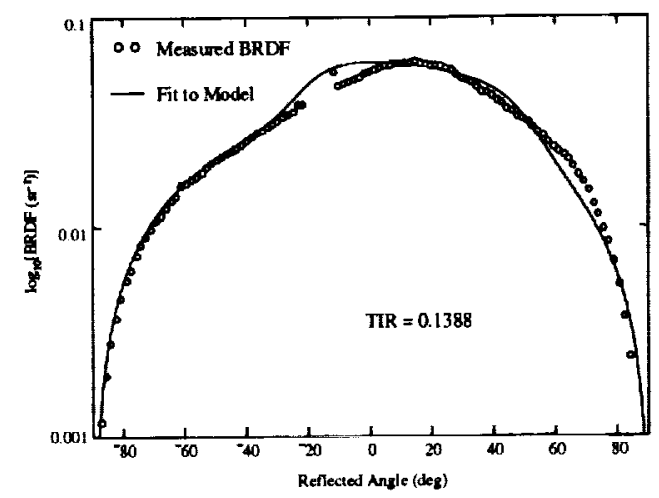

(b) Ceramic sample.

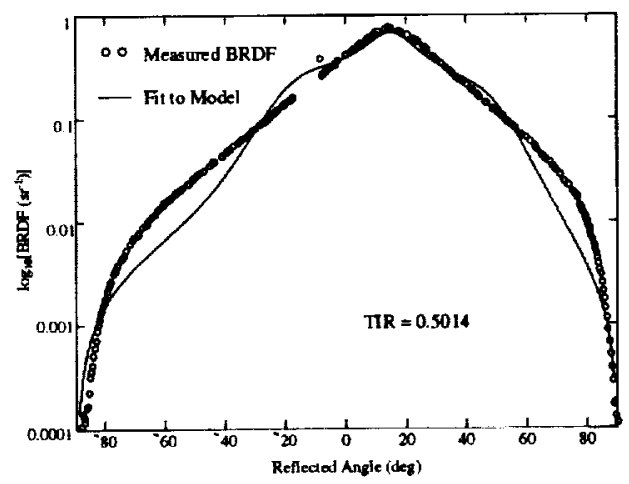

(d) Kovar sample.

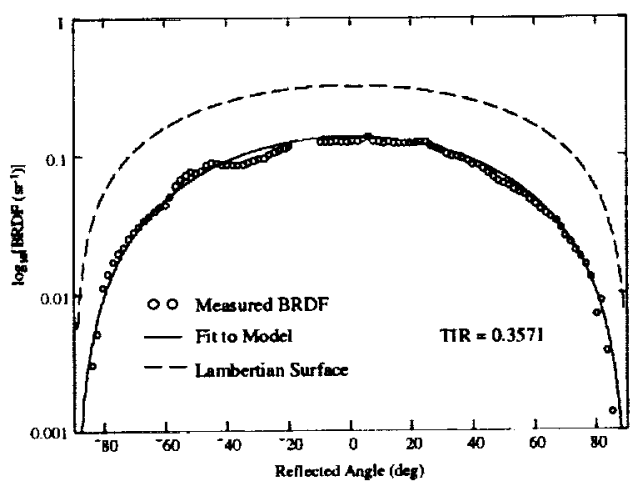

(f) Sandblasted aluminum sample.

Figure 5. BRDF measurements and empirical data fits.

Because $\mathrm{P}_{\text {Gauss }}$ and BRDF both vary across the surface, it is necessary to sum up contributions from the entire surface for each angle of reflection. Here we treat each area element as a point source with an angular dependent radiant intensity defined by $I_{r e f}$ at that location. It is important to note that each area element has a different $\theta_{i}, \theta_{r}, \theta_{\text {det }}$, and $R_{\text {refl. }}$. Because the planes are parallel, $\theta_{\text {det }}=\theta_{r}$ for each area element. Summing over all of the area elements $\left(\Delta \mathrm{a}_{\mathrm{ref}}\right)$, the irradiance at the plane of detectors is:

$$
E \approx \Delta a_{\text {refl }} \sum_{\mathrm{a}_{\mathrm{ref}}} \frac{\mathrm{I}_{\mathrm{refl}} \cos \theta_{\mathrm{det}}}{R_{\mathrm{refl}}^{2}}
$$

Combining all of the terms, a general expression for the irradiance is given by: 


$$
\mathrm{E}^{-}=2 \pi \mathrm{I}_{\mathrm{e}}\left[1-\cos \left(\frac{\Psi}{2}\right)\right] \Delta \mathrm{a}_{\text {refl }} \sum_{\mathrm{a}_{\mathrm{ren}}} \frac{\mathrm{P}_{\mathrm{Gauss}} \cdot \mathrm{BRDF} \cdot \cos \theta_{\text {del }}}{\mathrm{R}_{\mathrm{refl}}^{2}}
$$

where the detector is assumed to be planar.

This sum must be performed separately for each detector location in the plane of incidence. From Equation 9 it is apparent that the irradiance increases linearly with the radiant intensity from the emitter, and decreases quadratically with the distance between the planes. The magnitude also increases linearly with the reflectivity of the reflector surface. The distribution of the irradiance depends upon the beam profile of the emitter and the BRDF of the reflector surface.

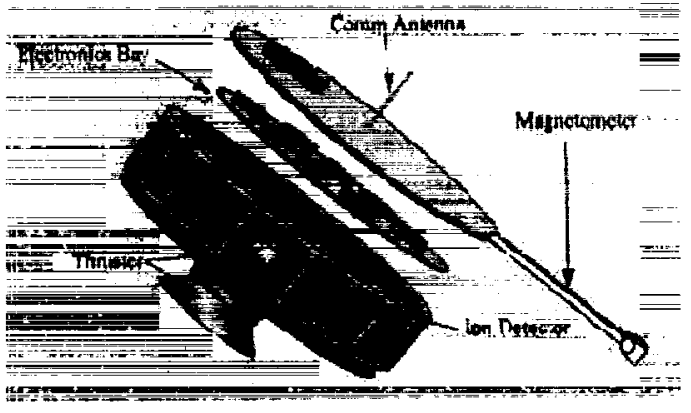

Figure 6. Example of a nanosatellite designed by NASAJGSFC.

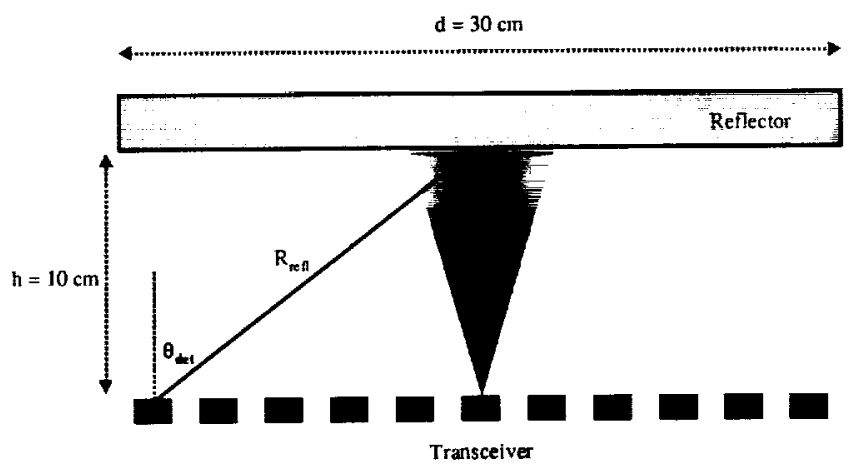

Figure 7. Geometry for nanosatellite IR communications model.

The parameters in Equation 8 can be varied to study the irradiance produced by different emitters, reflectors, and geometries. The Infrared Data Association (IrDA) provides guidelines for optical components [Ref. 3] that guarantee 4 $\mathrm{Mb} / \mathrm{sec}$ point-to-point data transmission from $0-1 \mathrm{~m}$ at with a maximum bit error rate of $10^{-8}$. Although our setup is somewhat different, we will use the IrDA specifications as a starting point for the analysis. The IrDA guidelines specify that each emitter must operate between 850 and $900 \mathrm{~nm}$, have a radiant intensity of 100 to $500 \mathrm{~mW} / \mathrm{sr}$, and have an emission angle between $15^{\circ}$ and $30^{\circ}$. Two infrared LEDs (Agilent HSDL-4220 and HSDL-4230) meet these criteria, and each is implemented in the model. The IrDA also requires that the irradiance received by each detector is between $10 \mu W / \mathrm{cm}^{2}$ and $500 \mathrm{~mW} / \mathrm{cm}^{2}$, which corresponds with a variation of $47 \mathrm{~dB}$.

Figure 8(a-f) compares the irradiance distribution from each reflector material in the plane of incidence for the HSDL-4230 emitter $\left(\mathrm{I}_{c}=375 \mathrm{~mW} / \mathrm{sr}, \psi=17^{\circ}\right)$. Figure 9(a-f) shows similar plots for the HSDL-4220 emitter $\left(\mathrm{I}_{c}=190\right.$ $\mathrm{mW} / \mathrm{sr}, \psi=30^{\circ}$ ). In Figures $8 \mathrm{f}$ and $9 \mathrm{f}$, the irradiance distribution from a sandblasted aluminum sample is compared with a perfectly Lambertian surface (indicated by the dashed line). In each figure, the dotted line denotes the minimum threshold level dictated by the IrDA protocol. Below this level the irradiance is too low to be detected by the photodiode. These results can be extended to the entire plane of detectors by noting that the reflectance is rotationally symmetric about the specular direction. Here $\theta_{\mathrm{r}}$ is defined as the angle between the optic axis of the emitter and the line-of-sight to the detector.

Figures 8 and 9 show that as the emission angle of the source increases, the range of reflected angles that receive sufficient irradiance also increases. This effect is most dramatic for samples with a strong specular component, such as a solar cell. In this case most of the irradiance is concentrated about a single angle of reflection, defined by $\theta_{\mathrm{r}}=-\theta_{\mathrm{i}}$. Changing the angle of incidence shifts the peak irradiance, and redistributes the irradiance about the corresponding angle of reflection. On the other hand, if the reflector is perfectly Lambertian, the irradiance is symmetrically distributed about the normal to the surface, regardless of the angle of incidence. For these two cases, the Lambertian surface provides sufficient irradiance over the largest range of reflected angles; however, the solar cell reflects a stronger signal at its peak reflection angle.

It is important to note that although the BRDF measurements were performed with a HeNe laser operating at 632.8 $\mathrm{nm}$, this model implements an LED at $875 \mathrm{~nm}$. Because the difference in wavelength is small, the reflectance characteristics are expected to be similar for the two cases. However, because infrared light has a longer wavelength, it is less sensitive to surface roughness, which means that the infrared BRDF will be slightly less diffuse than the visible BRDF. 


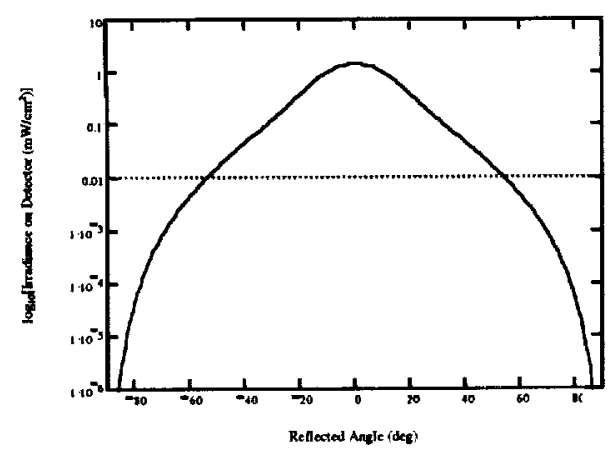

(a) Aluminum sample.

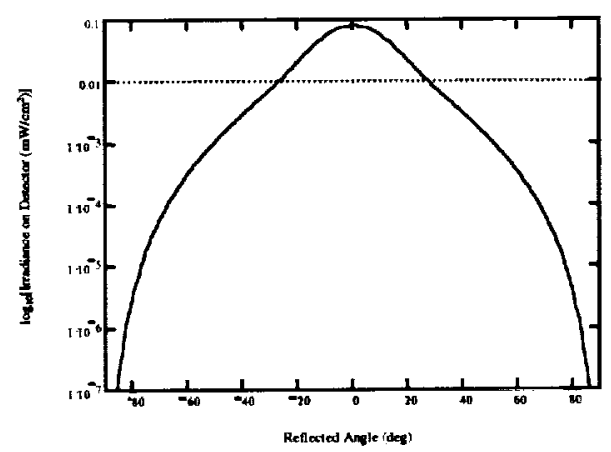

(c) Delrin sample.

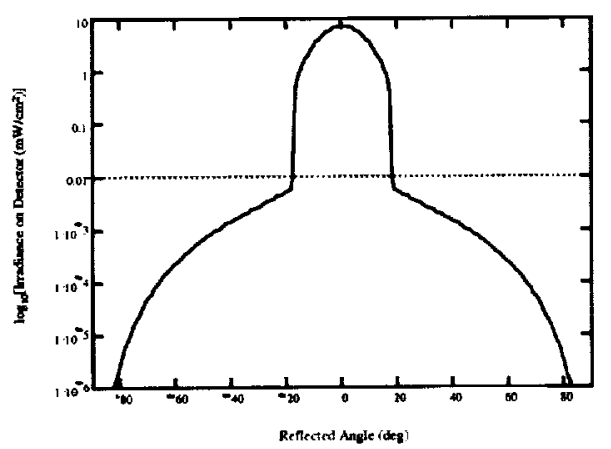

(e) Solar cell sample.

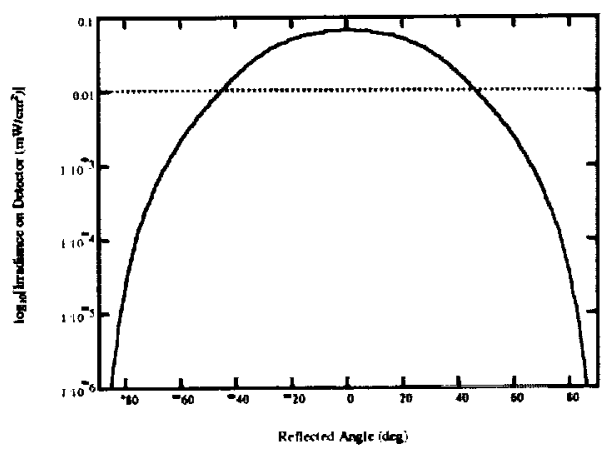

(b) Ceramic sample.

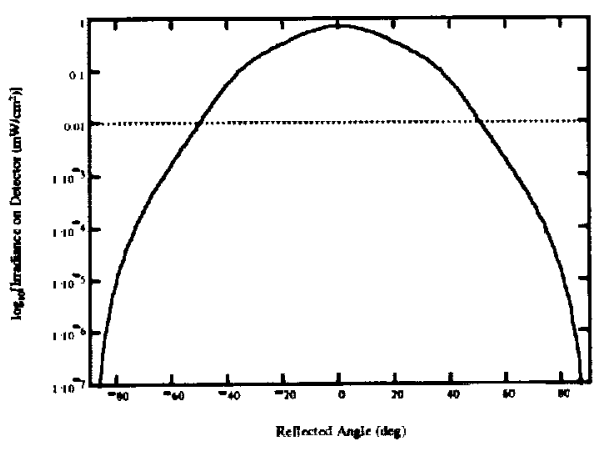

(d) Kovar sample.

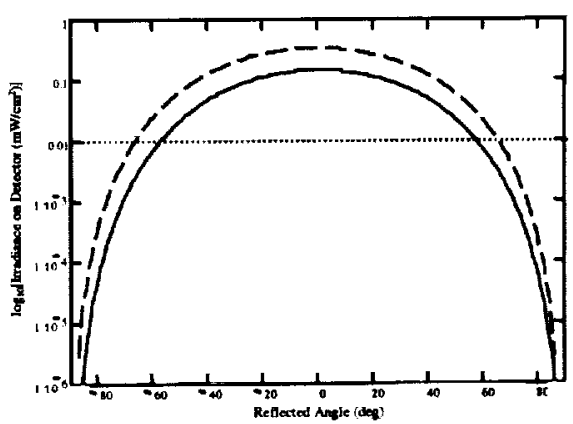

(f) Sandblasted aluminum sample.

Figure 8. Irradiance received by plane of detectors for HSDL-4230 emitter.

\subsection{Recommendations for Implementation}

For this rectangular geometry, in order to achieve the maximum irradiance over a large range of reflection angles, the reflecting surface should have a high reflectivity and be as nearly Lambertian as possible. For example, sandblasted aluminum has a nearly Lambertian reflectance; however, its TIR is only 0.3571 . This reduces the irradiance received by the detectors. Another promising material is beadblasted aluminum, which has not yet been tested. There is usually a tradeoff between radiant intensity and emission angle for the source. The distance between the plane of transceivers and the reflecting material should be minimized. However, the placement of detectors is then limited by the distribution of irradiance. The largest required angle of reflection is determined by the maximum distance between two detectors, according to:

$$
\theta_{r_{\max }}=\tan ^{-1}\left(\frac{d}{h}\right)
$$




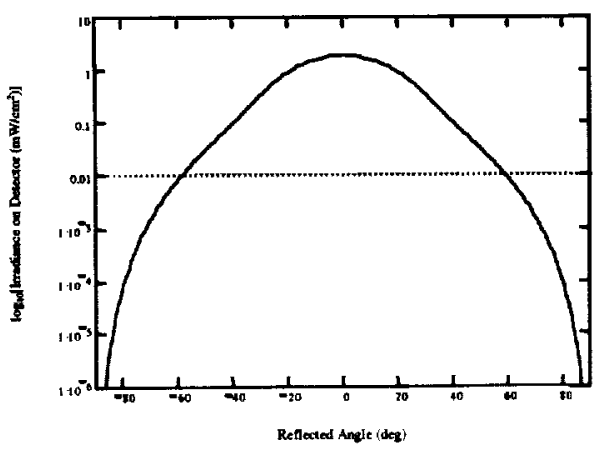

(a) Aluminum sample.

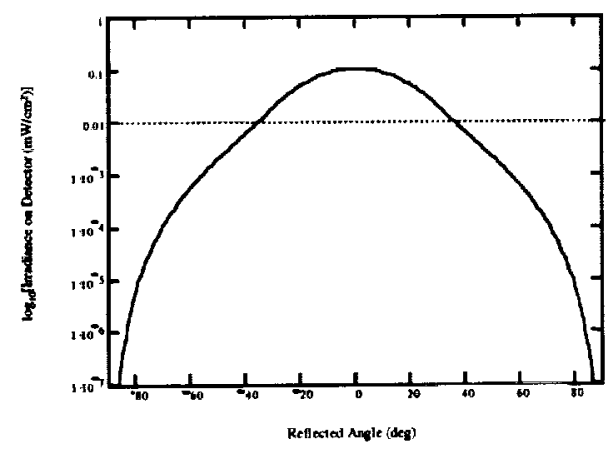

(c) Delrin sample.

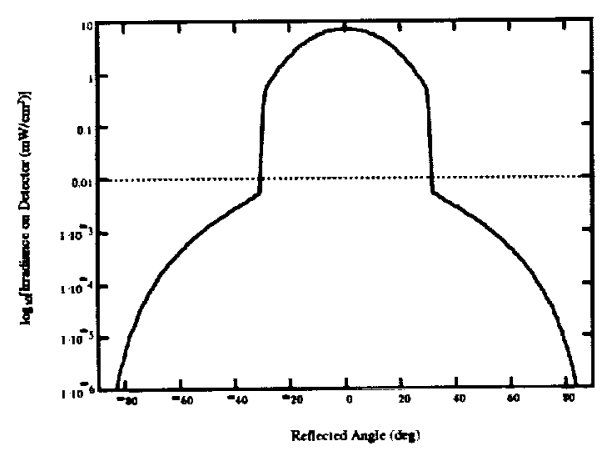

(e) Solar cell sample.

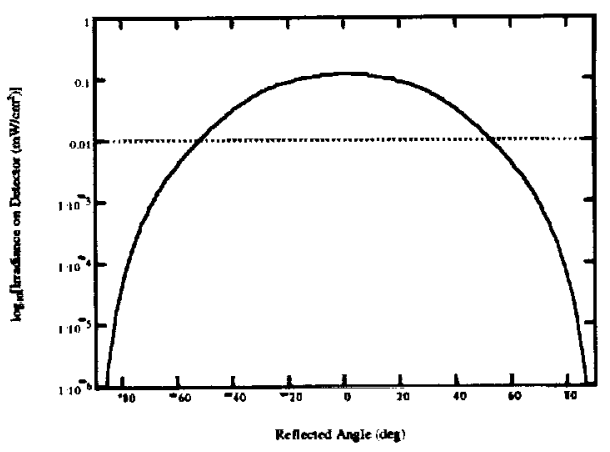

(b) Ceramic sample.

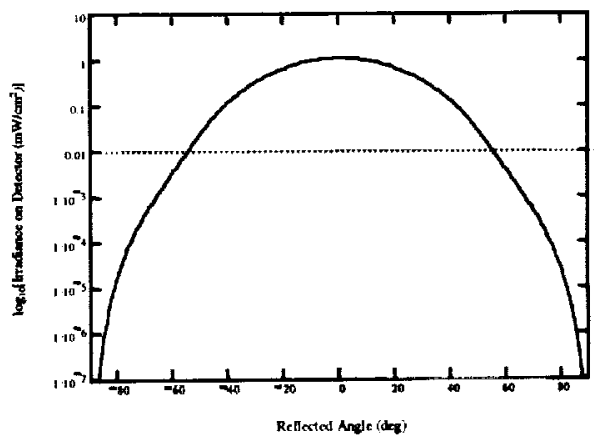

(d) Kovar sample.

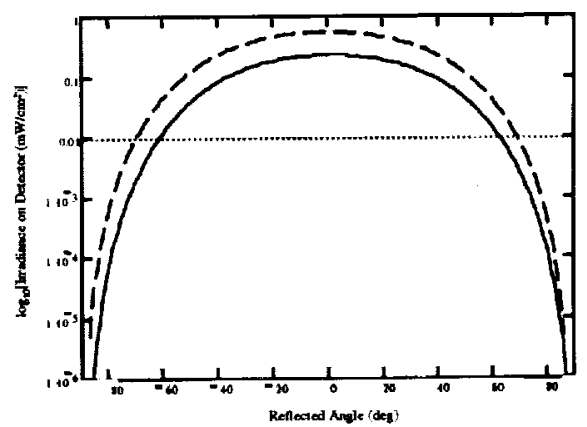

(f) Sandblasted aluminum sample.

Figure 9. Irradiance received by plane of detectors for HSDL-4220 emitter.

If $\mathrm{h}$ is fixed by the dimensions of the satellite, $\theta_{r_{\max }}$ can be decreased by shortening the distance between the most extreme detectors. The emitters and detectors can be purchased separately or together in a transceiver package. IrDA compliant transceiver modules (such as Agilent HSDL-1100) consist of a high-speed, high efficiency TS AIGaAs LED, a silicon PIN photodiode, and a bipolar, silicon integrated circuit, which contains an LED driver and a receiver providing two output signals with different data transmission rates. Also, a receiver lens magnifies the effective area of the detector to increase sensitivity.

The BRDF measurements and analysis described here could also be implemented into radiometric models for other geometries. For example, if the transceivers were placed along the interior vertical walls of the nanosatellite in Figure 6, the reflector would be a curved surface in the plane of incidence. This could increase the relative signal level received by outlying detectors. However, because the signal must travel a longer distance across the diameter of the satellite, the peak irradiance is expected to decrease. In order to increase the irradiance received by each detector, multiple emitters could be 
used to transmit each signal. By starting with the BRDF measurements, we can create models to represent various geometries, as determined by the physical layout of the satellite.

\subsection{Discussion of Link Margin}

Gfeller first proposed a non-directional IR wireless local area network using diffuse reflection [Ref. 4]. In geometries where a non-directive line-of-sight (los) configuration is possible, it is preferred over the diffuse link because it makes better use of the available power. In a spacecraft, however, we must assume that the internal link design is generally subordinate to the priority other spacecraft subsystems hold, i.e., they are considered the "users." As a result, internal spacecraft data links will probably have to contend with some obstacles to direct los communications. The best alternative in this case is to have diffuse reflections, preferably only one.

For sufficiently large detector FOVs, a hemispherical immersion lens on the photodetector offers the highest optical throughput, which, in the absence of any significant background light (within a satellite enclosure, where all external background sources can be essentially eliminated) is the best design for a IR free-space link that uses diffuse reflections for indirect line-of-sight (broadcast) communications. Within a confined enclosure, such as a cube with diffuse surfaces, the los link loss is much less than the non-los link loss, provided that the transmitter and receiver are relatively close together (with respect to the dimensions of the enclosure). However, when they are more separated, the losses are comparable. Typical link losses are in the range of -54 to $-74 \mathrm{~dB}$ for enclosures of several meters size.

Non-los signals also suffer more temporal dispersion from multipath reflections, thus reducing the effective channel bandwidth. Although multiple reflections may occur, by careful placement of sources and receivers, they can usually be reduced to just one needed reflection for most expected satellite geometries. Hence, we can apply our measured and modeled BRDF results to estimate the signal-to-noise ratio (SNR) at each possible receiver position. The advantage of using one diffuse reflection is that smaller FOVs can be used if desired, which generally reduces the dispersion and increases bandwidth. However, previous measurements and modeling [Ref. 5] have shown that for receivers with large FOVs, i.e., $90^{\circ}$, achievable bandwidths vary from $5-37 \mathrm{MHz}$ for enclosures with dimensions of several meters, well above the bandwidths needed for 1553 operation. In nanosatellite-scale enclosures, link (space) losses should also be relatively insignificant compared to los link angle-dependent losses. By employing only one diffuse reflection, the angular variation loss can also be reduced, i.e., a relatively narrow source beam divergence is broadened significantly by one diffuse surface. This suggests that if the received signal level around obstacles in the satellite are reduced too much in certain directions by reflection off unprepared surfaces, a single diffuse patch, perhaps specially prepared for that purpose, may be sufficient to alleviate the loss. By measuring the various surfaces, we have attempted to bound the degree of variation expected for unprepared surfaces. We have also included the BRDF of sandblasted aluminum as a baseline example of a "prepared" surface for comparison.

The estimated variation in received irradiance for the geometry previously described is 0.10 to $100 \mathrm{~W} / \mathrm{m}^{2}$. For signal shot noise-limited operation the signal-to-noise ratio (SNR) is given by:

$$
S N R=\frac{i_{s}^{2}}{i_{n}^{w}}=\frac{i_{x}^{2}}{2 e i_{s} B}=\frac{i_{s}}{4 e R_{b}}=\frac{E_{a v e} A_{\text {det }} R}{4 e R_{b}}
$$

where $i_{s}$ is the signal-induced photo-current, $i_{n}$ is the noise current, and $E_{\text {ave }}$ corresponds to the received irradiance required to support an estimate of the appropriate bit error rate (BER), where $R_{b}$ is the bit rate [bits per sec] necessary for on-off-keying (OOK) given a photodetector with area $A_{d e t}\left[\mathrm{~cm}^{2}\right]$ and responsivity $R[\mathrm{~A} / \mathrm{W}]$. The BER is then given by

$$
\mathrm{BER}=\operatorname{erfc} \sqrt{\mathrm{SNR}}
$$

where $\operatorname{erfc}(x)$ is the complementary error function. For a IrDA-compliant PIN photo-detector equivalent to that used in our system, the area is $\sim 10^{-3} \mathrm{~m}^{2}$, and the responsivity at the LED emission wavelength is $\sim 0.6 \mathrm{~A} / \mathrm{W}$. This yields a SNR of 4.7 $x 10^{5}$ for the lower bound on irradiance, compliant with the IrDA protocol $\left(0.01 \mathrm{~mW} / \mathrm{cm}^{2}\right)$. This guarantees a very low BER. It is likely, however, that interference from other sources of electrical noise at low frequencies (e.g., $60 \mathrm{~Hz}$ ) are more of a problem than optical sources within the satellite. 


\section{WIRELESS BUS IMPLEMENTATION}

\subsection{MIL-STD-1553 Summary}

MIL-STD-1553 was first issued in 1973 as a reliable solution to data sharing in complex avionics systems. The current version, 1553B, is required by all three services for inter- and intra-subsystem communication, and finds favor in the space community because of the availability of radiation hardened components. It is a dually redundant system designed for reliable communication among nodes having a fixed architecture. Typically, the bus controller (master) allows the chaining of multiple commands into major and minor time frames for performing tasks, such as data movement, independent of the host processor.

According to the MIL-STD-1553 standard [Ref. 6], communicating nodes at the physical layer may be directly or transformer coupled, may be voltage or current mode, and are always half-duplex. To provide DC isolation, they are typically transformer coupled (Fig. 10), requiring a zero average type of encoding such as Manchester II bi-phase level encoding. The bus is typically a copper bus consisting of twisted-shielded-pair wiring, with a $70 \mathrm{Ohm}$ impedance operating with $\pm 9 \mathrm{~V}$ to $\pm 13.5 \mathrm{~V}$ signals. The data bit clock is $1 \mathrm{MHz}$. This type of physical layer is generally quite power hungry.

At the data link layer, all command and data words are 20 clock cycles long, and consist of 3 clock cycles of sync bits, 16 clock cycles of data/command bits, and 1 cycle of odd parity bit. Sync signals are two 1.5 clock length pulses, whose polarity sequence identifies the transmission as command/status or data. There is only one active bus controller node (BC), up to 30 slave or remote terminal nodes (RT), and one broadcast node. All communication requests are initiated by one or more BC command. Data words and all non-broadcast RT responses are preceded by a status word. Both command and status sync bits are a 1-0 sequence and data is a 0-1 sequence. Any addressed RT must respond with a status word within less than $14 \mu \mathrm{sec}$ to be accepted as "alive".

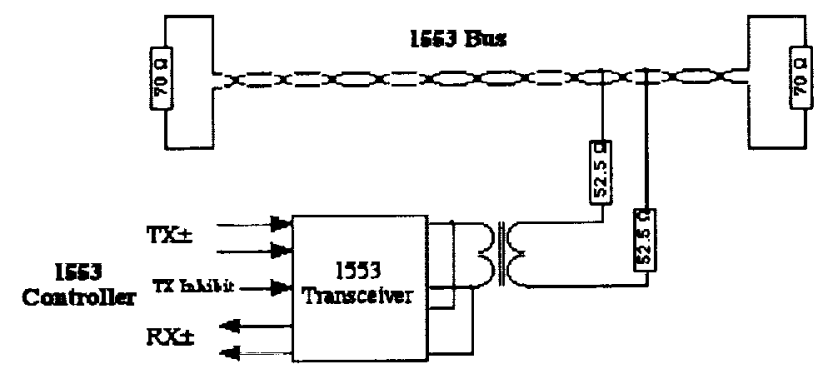

Figure 10. "Standard" MIL-STD-1553 Copper Bus and Transceiver

A typical 1553 transceiver chip, such as the BU-63152 [Ref. 7], provides separate complementary outputs and inputs to the 1553 controller, and performs a full-duplex to half-duplex conversion by gating the complementary output signals to the transformer primary voltage drivers whenever sending information. The primary transformer voltage is also level shifted and fed back to the complementary inputs to the controller so that the controller can verify that the primary drivers are working and that the 1553 copper bus is not shorted.

\subsection{IrDA Transceiver Summary}

The Infrared Data Association (IrDA) issued its standard for optical communication in 1994 [Ref. 8]. This interface was targeted at personal computer (PC) peripherals as a means for eliminating the peripheral cabling problems typically encountered with multiple peripherals connecting to one PC. The standard requires two speed ranges of operation. The low speed mode is used to establish link access after which the communicants can mutually establish the highest bit rate for reliable communication. The highest bit rate is $4 \mathrm{MHz}$ and the communication is limited to half-duplex.

The IrDA transceiver chip [Ref. 9] consists of an AC coupled LED for transmitting IR energy and an IR sensor with application specific post-processing. At its high-speed mode, it is optimized to work with $85-165 \mathrm{~ns}$ pulses in its receiver 
section. The transmitter section will not transmit pulses $>50 \mu \mathrm{s}$ in length. Of course, it is wise to use as short a pulse as possible for power conservation reasons. Signaling at a $4 \mathrm{MHz}$ rate occurs with $125 \mathrm{nsec}$ pulses using four-pulse position modulation. This modulation, however, requires phase-locking using a phasing preamble. One is not constrained to using this modulation scheme, however. An asynchronous unipolar return-to-zero (RZ) modulation scheme using 125 nsec pulses should also work.

The IrDA transceiver's application domain is to provide reliable communication over a variable range up to 1 meter in the presence of $10 \mathrm{~K}$ lux of sunlight, $1 \mathrm{~K}$ lux of fluorescent light, and $1 \mathrm{~K}$ lux of incandescent light. The transceiver thus incorporates automatic gain control circuitry for near/far communication distances with a settling time (Receive Latency) of $50 \mu \mathrm{sec}$ (maximum). The desirability of having the receive section operate while transmitting, for validating the operation of the transmitting LED, leads to a transmit to receive turn-around latency of $50 \mu \mathrm{sec}$ (maximum) to allow the receiver amplifier to recover from signal saturation.

\section{3 $\quad 1553$ to IrDA Transceiver Formatting}

Because the IrDA optimally senses $125 \mathrm{nsec}$ pulses in its $4 \mathrm{MHz}$ or fast IR (FIR) mode, the 1553 pulse train from the controller will be reformatted as shown in Figure 12 below. Starting at the rising edge of S0, it will be sampled by a 2 $\mathrm{MHz}$ clock and anytime the 1553 signal is high, a 125 ns pulse will be generated.

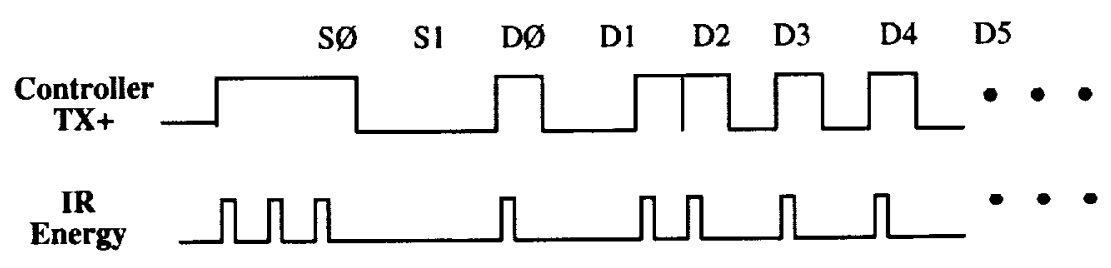

Figure 12. IR Encoding of 1553 pulse train

Since the IrDA transceiver will be receiving the transmission and since it is of a different format than the controller transmission, it cannot be used for wraparound verification of transmission. Thus it will be necessary to wraparound the

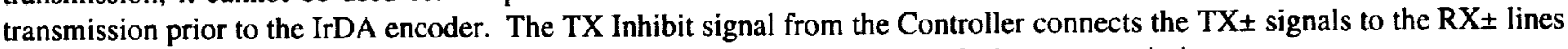
and disconnects the IrDA transceiver sensed output from the 1553 formatter during a transmission.

Reformatting the IrDA-received pulses to 1553 format consists of generating $500 \mathrm{nsec}$ pulses every time a $125 \mathrm{~ns}$ pulse is present. The total delay of formatting and reformatting would be at worst 1 sample interval as the transmit and receive clock are not phase locked and will exhibit a relative drift in phase. Figure 13 below shows the main functions needed to interface 1553 to $\operatorname{IrDA}$.

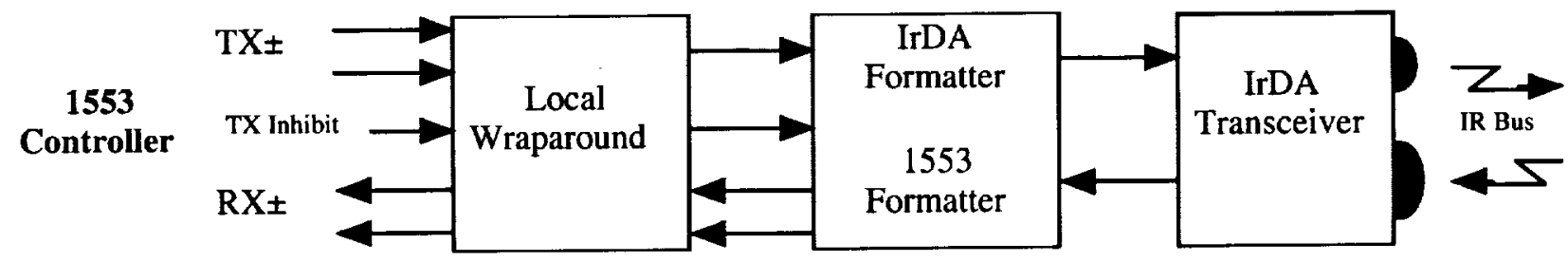

Figure 13. 1553 to IR conversion functional blocks.

\subsection{Power Comparison for the Two Physical Level Interfaces}

Although functionally the copper and IR interfaces should be the same, they differ in their power consumption and transmission distance performance. During the transmission of one word, the copper interface applies the full voltage (13.5 $\mathrm{V} \max$ ) across the $70 \Omega$ bus for a $100 \%$ duty cycle, due to its bipolar method of signaling. This energy constrained to the 
copper bus can communicate relatively long distances, limited only by cable losses and the response time requirement of $<14$ $\mu$ sec. According to Reference $7,1.7 \mathrm{~W}$ are required per transmitted word, with $550 \mathrm{~mW}$ required in quiescent mode.

The IR signaling method signals only during the high pulses (50\%), and then only for $125 \mathrm{nsec}$ of the $500 \mathrm{~ns}$ interval (25\%) for a total duty cycle of $12.5 \%$ per transmitted word. The light energy produced during the 125 nsec pulses is radiated into space resulting in a decrease in energy density $\left(\mu \mathrm{W} / \mathrm{mm}^{2}\right)$ received by the transceiver that is a function of many variables, as discussed previously. According to Reference 9, when operating the HSDL-3600 transceiver at $3.3 \mathrm{~V}$ it consumes $10 \mathrm{~mW}$ in standby and when operating at a peak pulse current of $650 \mathrm{~mA}$ consumes $650 \times 3.3 \times 0.125=270 \mathrm{~mW}$. Thus for direct viewing, IrDA transceivers placed $1 \mathrm{~m}$ apart, will consume $16 \%$ of the power consumed by a traditional 1553 copper bus. Of course, power consumption in the IrDA case can be trimmed for shorter communicating distances.

\subsection{3 to IrDA Format Conversion Board}

For demonstration purposes an IrDA transceiver PCB was built for a form-fit-function replacement of the 1553transceiver board that came with the MIL-STD 1553 BC/RT/MT PCMCIA Tester/Simulator Card purchased from Data Device Corporation. Figure 14 shows a pictorial comparison of the 1553 and IrDA cards.

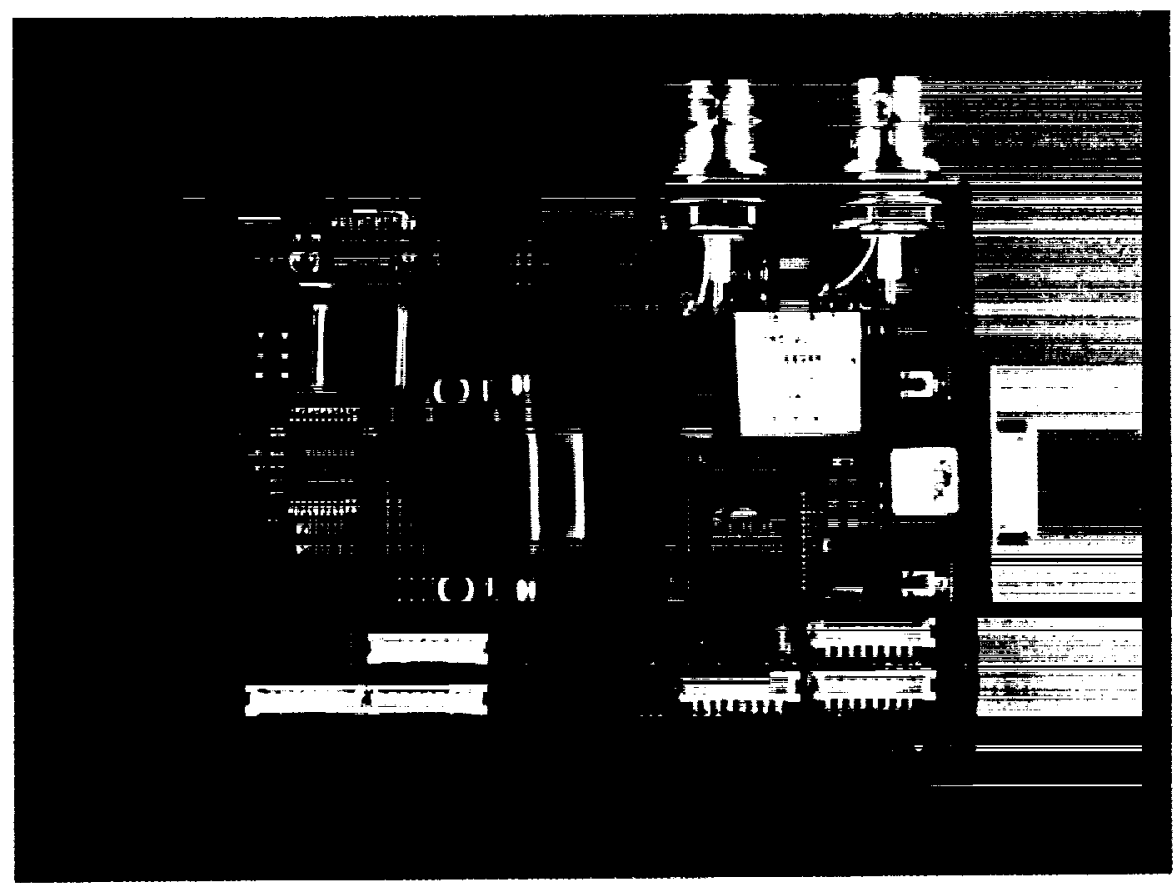

Figure 14. 1553 copper and IR transceiver boards

Figure 15 illustrates the schematic for the IrDA card. Local wraparound and formatting from 1553 to IrDA and vice versa are logical functions implemented in a Xilinx 9536 CPLD (Complex Programmable Logic Device). The 1553 transceiver is the HSDL-3600 from Agilent (formerly Hewlett Packard). Programming of the CPLD is performed via the JTAG interface.

\subsection{Applicability of IrDA/1553 Transceiver for Space Use}

The commercially available IrDA transceiver evaluated in the MIL-STD-1553 demonstration described in this section (the Agilent HSDL-3600) is a nicely integrated unit consisting of an IR LED, an IR sensor, and all of the necessary driving and postprocessing electronics. To our best knowledge, it has not been qualified for operation in a space environment with regard to materials, construction, and total dose, as well as single event radiation susceptibility. Furthermore, it has no 
built-in redundancy. These are limitations that emerged in recent testing of our unit. Furthermore, if used as part of the MILSTD-1553 protocol, the transceiver must be able to quickly switch between transmit and receive modes in order to meet the

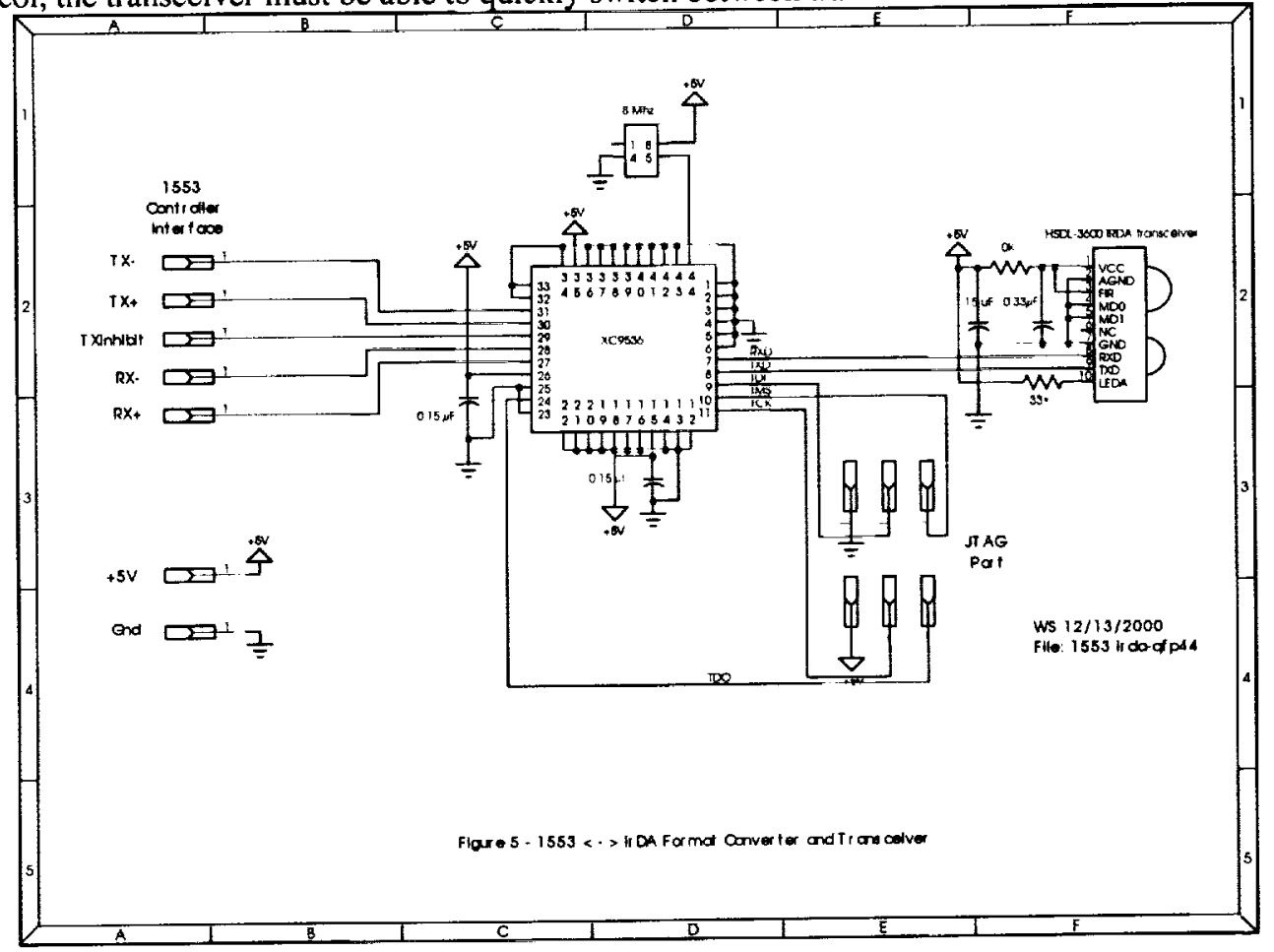

Figure 15. Schematic for the IrDA card.

$14 \mu \mathrm{sec}$ response time requirement. Although it is able to do this in the presence of strong signals, it is not able to meet this requirement for weak signals.

\section{CONCLUSIONS}

In summary, the demonstration unit successfully showed that the concept of IrDA/1553 communication link using diffuse IR reflections is possible, but has limitations that preclude its use at greater distances, because of limitations on delay $(14 \mu \mathrm{sec})$ and power (leading to higher than desired bit errors). This would be the case for satellite clusters. We will, however, be qualifying transceiver parts for this communication option and investigating extension to greater ranges.

Considering the expense and power requirements of one 1553 controller for each experiment in a microsatellite, other protocols may offer cost advantages. Many small microcomputers have an integrated UART (Universal Asynchronous Receiver Transmitter) running at speeds up to 115,200 Baud with several protocols to transfer data in an error free manner. Experiments can be interfaced in half duplex mode using differential or single ended signaling using the IrDA transceiver as is done with appropriately equipped PC peripherals. The IrDA transmit/receive turn-around time, however, can be up to 50 $\mu \mathrm{sec}$, which is greater than the 1553 response time requirement, and becomes problematic at lower power levels. Furthermore, it has no inherent redundancy as configured.

\section{ACKNOWLEDGEMENTS}

This work was performed for NASA under the Cross-Enterprise Technology Development Program.

\section{REFERENCES}

1. M.E. Thomas, "Measurement of Spectral Bidirectional Paint Reflectance and Determination of Spectral Directional Paint Emissivity," JHU/APL Technical Memorandum FIF(4)95-U-082, June 20, 1995. 
2. From Magnetospheric Constellation web site at NASA/Goddard Space Flight Center (http://sec.gsfc.nasa.gov/magcon_missionconcept.htm).

3. F.R. Gfeller, and U.H. Bapst, "Wireless In-House Data Communication via Diffuse Radiation," Proc. IEEE $\underline{67}, 1474$ (1979).

4. H. Hashemi, G. Yun, M. Kavehrad, F. Behbahani, and P. Galko, "Frequency Response Measurements of the Wireless Indoor Channel at Infrared Optics," Mobile Comm., Adv. Sys. and Comp., 1994 Int. Zurich Seminar on Dig. Comm. Proc., pp. 273-284, Zurich, 1994.

5. IrDA Data Link Design Guide, Hewlett Packard web site, www.semiconductor.agilent.com.

6. “MIL-STD-1553 Designer's Guide," Data Device Corporation.

7. "MIL-STD-1553 Data Bus Dual Transceiver," Data Device Corporation.

8. "SIR - Physical Layer Specification," www.irda.org.

9. "IrDA Compliant $4 \mathrm{Mb} / \mathrm{s} 3 \mathrm{~V}$ Infrared Transceiver," Agilent Technical Data Sheet. 